\title{
Half-Duplex Estimate-and-Forward Relaying: Bounds and Code Design
}

\author{
Arnab Chakrabarti, Alexandre de Baynast, Ashutosh Sabharwal, and Behnaam Aazhang \\ Department of Electrical and Computer Engineering \\ Rice University, Houston, Texas 77005 \\ Email: $\{$ arnychak,debaynas,ashu,aaz $\}$ rice.edu
}

\begin{abstract}
We propose a coding scheme for half-duplex estimate-and-forward relaying. Our first contribution is to present an achievable rate for the estimate-and-forward protocol. We also derive the achievable rate for the special case of a Gaussian half-duplex relay channel. Guided by the information theoretic coding scheme for the aforementioned achievable rate, we propose a practical coding scheme. The proposed coding scheme incorporates several design choices to reduce receiver complexity without compromising performance. Binary LDPC codes are used in the source broadcast phase. Estimation is performed by scalar quantization of the received signal at the relay. Finally, a procedure similar to maximal ratio combining is used to aggregate direct and relayed signals at the destination. An important practical advantage of our scheme is that it does not require symbol level source-relay synchronization. The codes outperform direct and two-hop channel capacities, as well as decode-and-forward relaying when the relay-destination link is strong.
\end{abstract}

\section{INTRODUCTION}

The last decade has seen an explosion of interest in usercooperation and relaying. Motivated by the gains promised by relaying, several researchers have investigated the design of coding schemes for the relay channel. To date, however, most of these efforts have concentrated on the decode-andforward relay protocol. In contrast, we propose achievable rates and code design for half-duplex estimate-and-forward relaying, which can outperform decode-and-forward relaying when the relay-destination link is strong.

Our first contribution is to present an achievable rate for half-duplex estimate-and-forward relaying. Assuming that the quantization noise is Gaussian, we also derive the achievable rate for the special case of a Gaussian relay channel. Estimateand-forward relaying is shown to yield a substantially higher rate than both direct and two-hop communication at low SNR when the relay is physically close to the destination.

Guided by the information theoretic random coding scheme for the aforementioned achievable rate, we propose a practical coding scheme for estimate-and-forward relaying. We incorporate several key ideas in our design to reduce receiver complexity. First, noting that the relaying gain is substantial only at low SNRs, we advocate the use of binary modulation over source-relay (SR) and source-destination (SD) links. This enables the use of capacity-approaching binary LDPC codes over these links, and also permits belief propagation decoding. The estimate forwarded from the relay to the destination is obtained through scalar quantization of the relay received signal.
The bin index obtained through scalar quantization is encoded and communicated error-free over the relay-destination (RD) link. Unlike the SR and SD links, we require higher order modulation to achieve the capacity of the strong RD link. The direct and relayed signals are combined using a procedure similar to maximal ratio combining, but which takes into account quantization noise in addition to channel noise.

The proposed coding scheme has several useful properties. First, recognizing the difficulty of performing shielding and accurate interference cancellation at the relay, we focus on half-duplex relaying. Another vital point is that the proposed coding scheme does not require symbol level synchronization between the source and the relay at any stage. Third, the observation that binary modulation over SR and SD links leads to negligible rate loss, allows the use of capacity-approaching LDPC codes. Although the RD link requires higher order modulation, we show that the overall achievable rate is robust to small changes in the rate of this link. Finally, our work demonstrates the efficacy of scalar quantization as a technique to perform estimation.

The three-terminal relay channel (depicted in Fig. 1) was introduced in [1]. The capacity of the general relay channel is still unknown, but the bounds discovered by van der Meulen were improved significantly in [2]. Two important relaying strategies were introduced in [2], which are now known as the decode-and-forward and estimate-and-forward relay protocols. Although early results on relaying focused exclusively on the full-duplex relay channel, the idea of half-duplex relaying has become popular in recent years due to its practicality, and a lot of research is now based on this model [3], [4], [5], [6]. With significant advances in technology, the promise of relaying is very real. Several research efforts are currently geared towards developing practical user-cooperation schemes to harvest the gains predicted by information theory. Solutions in this direction include [7], [8], [9], [10], [11], [12], [13], [14], [15], [16].

The rest of this paper is organized as follows. Section II describes the system model. Following that, in Section III we discuss information theoretic results on estimate-and-forward relaying. Section IV presents details of the proposed coding scheme. We present numerical results in Section V and finally conclude in Section VI. 


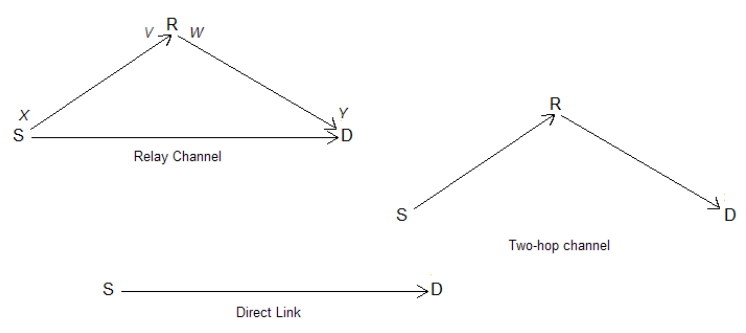

Fig. 1. Direct, two-hop and relay channels.

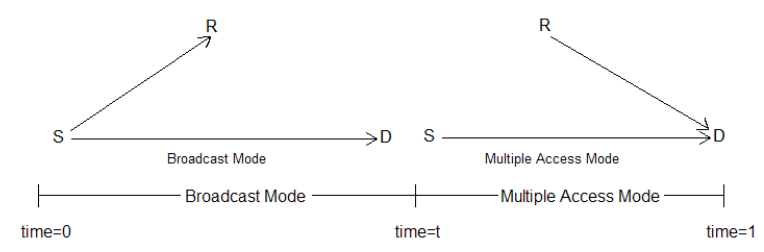

Fig. 2. Half-duplex relay modes.

\section{SYSTEM DESCRIPTION}

The relay channel is shown in Fig. 1. The source (S) sends data to the destination (D), aided by the relay (R). In the case of a half-duplex relay channel, the relay cannot transmit and receive simultaneously in the same band. Communication takes place over two slots of time fractions $t$ and $(1-t)$. In the first slot, $S$ transmits information, which is received by $\mathrm{R}$ and $\mathrm{D}$. We will call this the $\mathrm{BC}$ (broadcast) mode of communication. In the second slot, both $\mathrm{S}$ and $\mathrm{R}$ transmit information to D. We will refer to this as the MAC (multiple access) mode. These two modes are depicted in Fig. 2.

We compare half-duplex relaying with both direct and twohop communication (see Fig. 1). For fair comparison, the middle node is assumed to operate in a half-duplex fashion in the two-hop channel. In the context of a two-hop channel also, we use the terms BC and MAC mode to denote the phases in which the relay receives and transmits respectively.

We normalize transmitted power with noise power, which is assumed to be the same at each receiver. An average global transmission power constraint is imposed on the nodes, given by

$$
t P_{S_{B C}}+(1-t)\left(P_{S_{M A C}}+P_{R_{M A C}}\right) \leq P
$$

where $P$ represents the total system transmission power, ${ }^{1}$ $P_{S_{B C}}$ is source transmission power in BC mode, $P_{S_{M A C}}$ is source transmission power in MAC mode, and $P_{R_{M A C}}$ is relay transmission power in MAC mode. In the two-hop case, the same power constraint holds with $P_{S_{M A C}}=0$. For the direct link, the above power constraint is true with $t=1$.

We present information theoretic results under the standard assumption that the channel is represented by the conditional probability of the outputs given the inputs. We use $X, V, W$ and $Y$ to denote the source transmitted signal, the relay received signal, the relay transmitted signal, and the destination

\footnotetext{
${ }^{1} P$ is also the equivalent relay channel SNR in our plots.
}

received signal respectively (see Fig. 1). $\widehat{V}$ denotes an estimate of $V$. In the context of half-duplex relaying, subscripts 1 and 2 in conjunction with the channel inputs and outputs denote $\mathrm{BC}$ and MAC modes respectively.

For the purpose of code construction, we focus on onedimensional AWGN channels. ${ }^{2}$ The distance between $\mathrm{S}$ and $\mathrm{D}$ is normalized to unity, and $\mathrm{R}$ is assumed to lie on the straight line joining $\mathrm{S}$ and $\mathrm{D}$. The relay position, denoted $d$, represents its distance from the source. The collinearity of S,R and D is not used in deriving any of our results, but it enables a simple one-dimensional characterization of the relay position. The SD channel gain is given by $\gamma_{S D}=1$, the SR gain is $\gamma_{S R}=\frac{1}{d^{\lambda}}$, and the RD gain is $\gamma_{R D}=\frac{1}{(1-d)^{\lambda}}$, where $\lambda$ is the channel attenuation exponent. Using the above notation, the Gaussian half-duplex relay channel model is as follows

$$
\begin{aligned}
Y_{1} & =\gamma_{S D} X_{1}+n_{1} \\
V_{1} & =\gamma_{S R} X_{1}+n_{2} \\
\widehat{V_{1}} & =V_{1}+n_{q} \\
Y_{2} & =\gamma_{S D} X_{2}+\gamma_{R D} W_{2}+n_{3}
\end{aligned}
$$

where

$$
\begin{aligned}
E\left[X_{1}^{2}\right] & =P_{S_{B C}} \\
E\left[X_{2}^{2}\right] & =P_{S_{M A C}} \\
E\left[W_{2}^{2}\right] & =P_{R_{M A C}}
\end{aligned}
$$

satisfy (1). Here $n_{1}, n_{2}$, and $n_{3}$ are all zero mean Gaussians with variance $\sigma^{2}$. We model the estimate as a quantized version of the relay received signal, and $n_{q}$ represents the quantization noise, which we assume to be zero-mean Gaussian with variance $\sigma_{q}^{2}$.

\section{INFORMATION THEORETIC RESULTS ON ESTIMATE-AND-FORWARD RELAYING}

The following theorem from [2] gives the achievable rate of full-duplex estimate-and-forward relaying

Theorem 3.1: [2] The rate $R_{f d}$ is achievable for a fullduplex relay channel where

$$
R_{f d}=\sup I(X ; Y, \widehat{V} \mid W)
$$

subject to the constraint

$$
I(W ; Y) \geq I(V ; \widehat{V} \mid W, Y)
$$

where the supremum is over all joint distributions of the form $p(x, w, y, v, \widehat{v})=p(x) p(w) p(y, v \mid x, w) p(\widehat{v} \mid v, w)$ on $\mathcal{X} \times \mathcal{W} \times$ $\mathcal{Y} \times \mathcal{V} \times \widehat{\mathcal{V}}$ and $\widehat{V}$ has a finite range.

The above theorem introduces the idea of an estimate $\widehat{V}$ of $V$ that is communicated reliably from $\mathrm{R}$ to $\mathrm{D}$ when decoding is not possible. For half-duplex estimate-and-forward relaying, we derive the following theorem.

Theorem 3.2: The following rate is achievable for a halfduplex relay channel

$$
R_{h d}=\sup _{0 \leq t \leq 1} t I\left(X_{1} ; Y_{1}, \widehat{V_{1}}\right)+(1-t) R_{1}
$$

\footnotetext{
${ }^{2}$ An extension to circularly symmetric AWGN is straightforward.
} 
subject to the constraint

$$
t I\left(V_{1} ; \widehat{V_{1}} \mid Y_{1}\right) \leq(1-t) R_{2}
$$

where $R_{1}$ and $R_{2}$ satisfy

$$
\begin{aligned}
R_{1} & \leq I\left(X_{2} ; Y_{2} \mid W_{2}\right) \\
R_{2} & \leq I\left(W_{2} ; Y_{2} \mid X_{2}\right) \\
R_{1}+R_{2} & \leq I\left(X_{2}, W_{2} ; Y_{2}\right)
\end{aligned}
$$

We describe the encoding and decoding, and sketch an outline of the proof.

The information can be conceptually separated into two components $(P, Q)$. In time-division half-duplex relaying, communication takes place in two time slots, corresponding to the broadcast (BC) and multiple-access (MAC) modes of communication. In BC mode, the source encodes information $P$ to generate a codeword $X_{1}$ for transmission, which is received corrupted as $Y_{1}$ by the destination and as $V_{1}$ by the relay. Neither the destination, nor the relay attempts to decode this information. The destination stores its received signal, whereas the relay generates an estimate (a compressed or quantized signal) $\widehat{V}_{1}$ from $V_{1}$ and using knowledge of the distribution of $Y_{1}$. In the second (multiple-access) phase, the relay encodes its compressed signal $\widehat{V_{1}}$, to yield a codeword $W$ for transmission over the RD link. The compression of $V_{1}$ is at a rate $R_{2}$ such that the capacity of the RD link permits reliable transmission of the compressed signal $\widehat{V_{1}}$. In the second phase, the source transmits new information $Q$ at a rate $R_{1}$ decodable by the destination. We outline the proof as follows.

In $\mathrm{BC}$ mode, we may think of $\mathrm{S}$ as a single transmitter and $\mathrm{R}$ and $\mathrm{D}$ as a pair of receivers that communicate over the RD link. This resembles a $1 \times 2$ MIMO system. If lossless communication over the RD link were possible, then the capacity of this $1 \times 2$ channel would be $I\left(X_{1} ; Y_{1}, V_{1}\right)$. However, since the RD link can communicate only $\widehat{V_{1}}$ reliably (by condition (12)), the rate we can achieve is limited to $I\left(X_{1} ; Y_{1}, \widehat{V_{1}}\right)$ in the $\mathrm{BC}$ mode. In MAC mode, $\mathrm{S}$ and $\mathrm{R}$ act as sources, and $\mathrm{D}$ as the destination. This is a multiple-access channel. R sends $\widehat{V_{1}}$ to $\mathrm{D}$ over the RD link at a rate $R_{2}$. The remaining capacity of the MAC is utilized by $\mathrm{S}$ to send new information $Q$ to the destination at a rate $R_{1}$. The rates $R_{1}$ and $R_{2}$ belong to the capacity region of the multiple-access channel.

Corollary 3.3: The rates $R_{1}=I\left(X_{2} ; Y_{2}\right)$ and $R_{2}=$ $I\left(W_{2} ; Y_{2} \mid X_{2}\right)$ satisfy (13).

We will use the achievable rate corresponding to the above corollary for code design. There are other achievable rates corresponding to amplify and forward and decode and forward protocols, but we will not consider them here. The authors have proposed LDPC code designs for decode-and-forward relaying in [11].

\section{A. Achievable rates for Gaussian channels}

Let $C(x)$ denote the function $\frac{1}{2} \ln (1+x)$. Assuming all input distributions to be Gaussian, we derive the following expressions.

$$
\begin{aligned}
I\left(X_{1} ; Y_{1}, \widehat{V_{1}}\right) & =C\left(1+\frac{\gamma_{S D}^{2} P_{S_{B C}}}{\sigma^{2}}+\frac{\gamma_{S R}^{2} P_{S_{B C}}}{\sigma^{2}+\sigma_{q}^{2}}\right) \\
I\left(X_{2} ; Y_{2}\right) & =C\left(1+\frac{\gamma_{S D}^{2} P_{S_{M A C}}}{\gamma_{R D}^{2} P_{R_{M A C}}+\sigma^{2}}\right) \\
I\left(W_{2} ; Y_{2} \mid X_{2}\right) & =C\left(1+\frac{\gamma_{R D}^{2} P_{R_{M A C}}}{\sigma^{2}}\right) \\
I\left(V_{1} ; \widehat{V_{1}} \mid Y_{1}\right) & =H\left(\widehat{V_{1}} \mid Y_{1}\right)-H\left(\widehat{V_{1}} \mid V_{1}, Y_{1}\right) \\
& =H\left(\widehat{V_{1}} \mid Y_{1}\right)-H\left(\widehat{V_{1}} \mid V_{1}\right) \\
& =H\left(\widehat{V_{1}}, Y_{1}\right)-H\left(Y_{1}\right)-H\left(\widehat{V_{1}} \mid V_{1}\right) \\
& =C\left(1+\frac{\sigma^{2}}{\sigma_{q}^{2}}\left(1+\frac{\gamma_{S R}^{2} P_{S_{B C}}}{\sigma^{2}+\gamma_{S D}^{2} P_{S_{B C}}}\right)\right)
\end{aligned}
$$

Fig. 3 shows the achievable rate of estimate-and-forward relaying and compares it with those of direct and two-hop communication over AWGN channels. For relay position $d=$ 0.95 and channel attenuation $\lambda=3$, the throughput increase over both direct and two-hop relaying is in excess of $50 \%$ at low SNRs. Note that the rate of estimate-and-forward relaying as a multiple of the direct link capacity approaches 1 at high SNRs. Therefore, the relaying gain is significant only at low SNRs, a result also known to be true for decode-and-forward relaying [17], [11]. The achievable rate can be expected to increase and approach the capacity of the $1 \times 2$ SIMO channel as $d \rightarrow 1$.

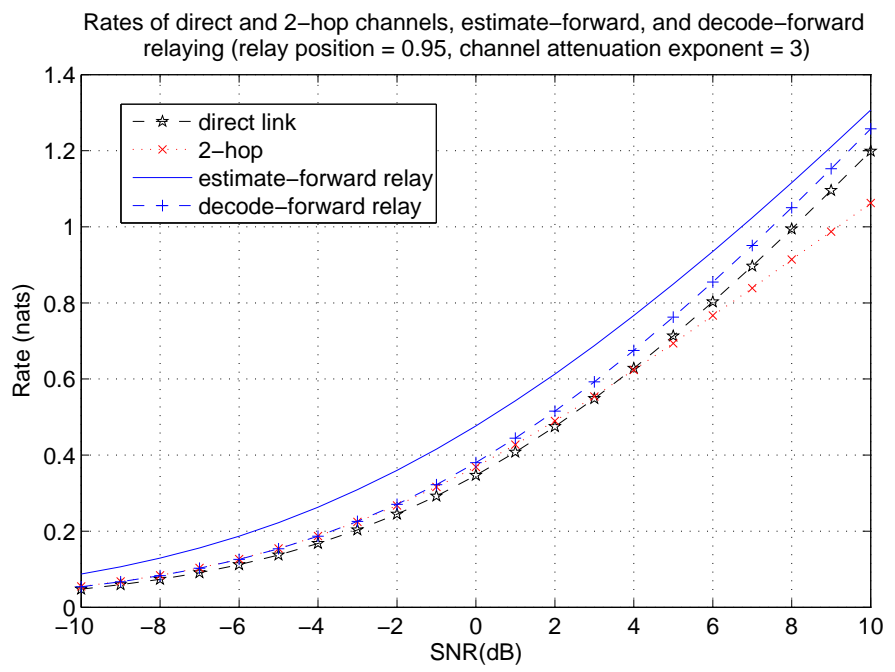

Fig. 3. Performance of estimate-and-forward relaying over AWGN channels.

\section{LDPC CODE DESIGN}

The proposed coding scheme is inspired by the information theoretic results derived in Section III. In order to reduce receiver complexity at both the relay and the destination, we take advantage of several facts.

First, as noted before, the gain of estimate-and-forward relaying is maximum at low SNRs, where binary modulation is capable of approaching capacity. We exploit this fact to 
reduce decoding complexity by using binary modulation for the source broadcast in $\mathrm{BC}$ mode. An additional advantage of doing so is that we can use binary LDPC codes that have been specifically optimized for the binary AWGN channel, and achieve near-capacity performance. The use of binary LDPC codes for source broadcast is one of the cornerstones of our code design.

Given a received signal at the relay, the all-important question is: how should we form the estimate? As mentioned before, we limit our attention to quantization for forming the relay estimate. Further, we focus only on scalar quantization. It has been proved in [18] that when the quantization rate is low, which is true in our case, the performance of scalar quantization asymptotically approaches that of vector quantization. This is the second key feature of our coding scheme.

We use a combination of scalar quantization and source coding to approach the entropy of the estimate. An extended discussion of this idea can be found in Chapter 9 of [19]. A short description of the particular quantization scheme used in this paper will follow in Section IV-A.

The quantized signal at the relay is then channel coded and communicated over the RD link. Note that, unlike the SR and SD links, the RD link must be strong for estimateand-forward relaying to be beneficial. For a strong RD link, binary modulation can only achieve a fraction of its capacity. Therefore, we will use higher-order modulation for the RD link.

The third key design choice that we make is to allocate all of the MAC mode power to the relay. In other words, the source is silent in MAC mode. This choice is motivated by numerical power allocation results. For a wide range of relay positions and SNRs, we have consistently found that the achievable rate is maximized when all of the MAC mode power is allocated to the relay. This last choice dramatically simplifies receiver design because communication in the MAC mode is now singleuser communication. The relay signal can be decoded without successive interference cancellation, which would normally be required in a multiple-access channel. Even more importantly, note that as a consequence of the above simplification, the source and the relay transmit in different time slots, thereby eliminating the need for source-relay symbol synchronization. In terms of practical implementability, this last property gives a tremendous advantage to the proposed scheme, since synchronization between transmitters in a multiple-access channel is difficult, and is rarely implemented in practice.

Finally, the BC and MAC mode received signals are combined and decoded at the destination. The destination first decodes the MAC mode signal from the relay, and uses it to reconstruct the quantized version of the relay received signal. Then it performs maximal ratio combining of the source and quantized relay signals, keeping in mind that the noise in the quantized relay signal is an aggregate of quantization noise and channel noise over the SR link. Details of maximal ratio combining can be found in [20].

\section{A. Scalar Quantizer Design}

We describe the design of our quantizer as follows. Let $n_{b}$ be the number of bits available to quantize each received symbol at the relay. The probability density function of a received symbol at the relay is called $d$. For binary modulation over the SR link, $d$ is a combination of two Gaussians with different means but the same variance as shown in Fig. 4. The quantizer uses $N-1$ thresholds to partition $d$ into $N$ sets with probability $p_{1}, p_{2}, \ldots, p_{N}$, where $N$ is determined by $n_{b}$ as described below. The following properties define the quantizer used by us.

1) $H\left(p_{1}, p_{2}, \ldots, p_{N}\right)=n_{b}$,

2) $N=\left\lceil 2^{n_{b}}\right\rceil$, i.e. the number of quantizer levels (or sets) is no more than the minimum number necessary to meet the entropy constraint,

3) the thresholds are placed symmetrically with respect to the origin,

4) among quantizers that meet all three of the above constraints, we choose the one that is best with respect to the absolute difference metric. The reason for the choice of this distortion measure is that the log likelihood ratio, which is the input to the Belief Propagation algorithm at the decoder, is a quantity proportional to the channel output. Therefore, the absolute difference of channel outputs captures the impact of quantization noise on decoder performance.

Fig. 4 shows an example of quantizing the relay signal when $1<n_{b} \leq \log _{2} 3$.

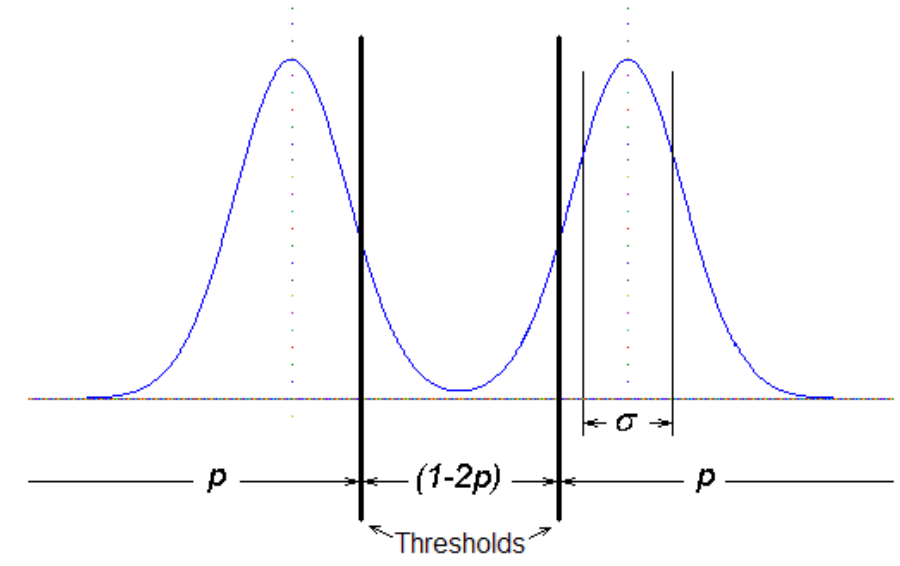

Fig. 4. Quantization example: Partitioning of the relay received signal into three sets.

\section{NUMERICAL RESULTS}

In this section, we present numerical results for the relay coding scheme. The LDPC code blocklength for the simulations is $100 \mathrm{~K}$, and the degree distributions are obtained from [21]. We do not explicitly simulate coding over the RD link, and use its capacity as its rate. However, this does not 
compromise accuracy since the overall achievable rate is not sensitive to small changes in the RD link rate at low SNR. This is shown in Fig. 5, which plots the relay achievable rate when different fractions of the the RD link capacity are achieved.

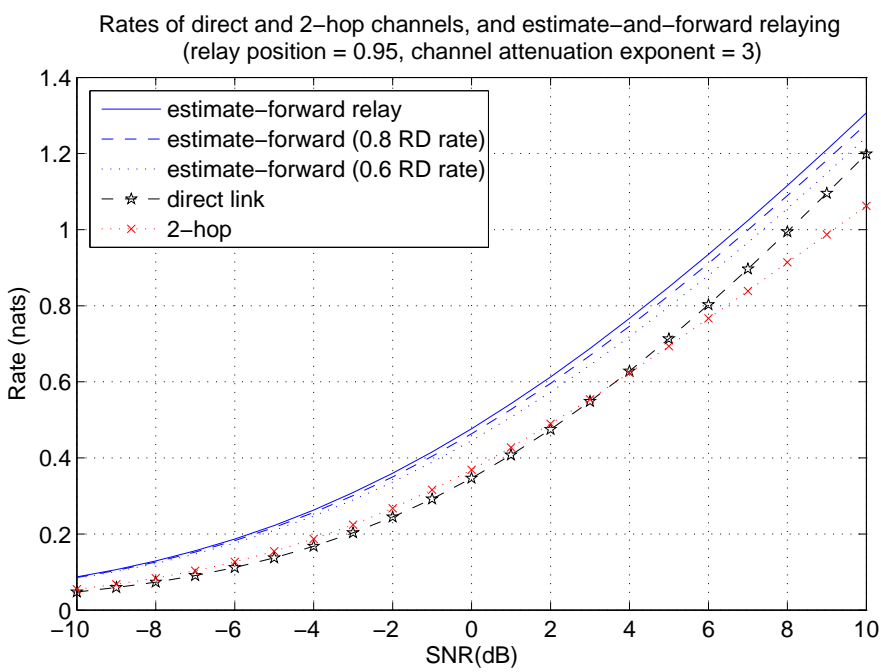

Fig. 5. Rate of estimate-and-forward relaying when RD link achieves a fraction of its capacity.

The performance of the proposed coding scheme is shown in Fig. 6. For the codes, we plot the achievable rate of the code, vs. the SNR at which the BER becomes sufficiently small. The codes outperform the capacities of both direct and two-hop channels, as well as decode-and-forward relaying. Note that here we are comparing practical codes for the relay channel with theoretical limits for the other two channels. The margin of gain would be larger if we compared relay codes with actual codes for direct and two-hop communication.

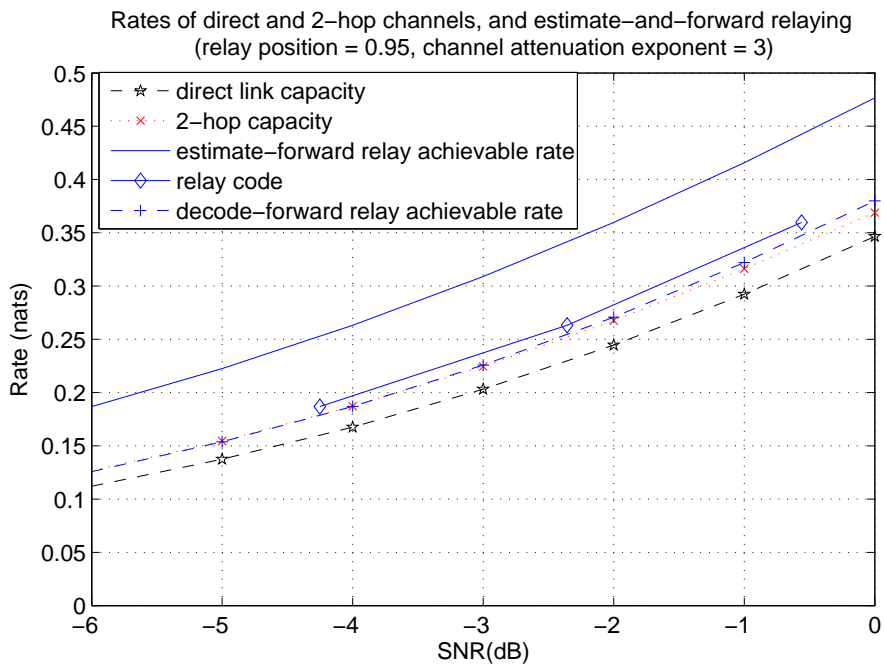

Fig. 6. Performance of the relay coding scheme.

\section{CONCLUSIONS AND FUTURE DIRECTIONS}

We have outlined an implementation for half-duplex estimate-and-forward relaying. The proposed coding scheme outperforms direct and two-hop communications. Our current focus is on defining suitable distortion metrics, and designing a provably good quantization strategy. Whether or not there exists a low-complexity vector quantization scheme is yet another open question. In another direction, the proposed scheme can be extended to fading channels, and to scenarios where accurate channel state information is not available at the transmitters.

\section{REFERENCES}

[1] E.C. van der Meulen, "Three-terminal communication channels," $A d$ vanced Applied Probability, vol. 3, pp. 120-154, 1971.

[2] T. M. Cover and A. A. El Gamal, "Capacity theorems for the relay channel," IEEE Trans. Inform. Theory, vol. 25, pp. 572-584, Sep. 1979.

[3] M.A. Khojastepour, A. Sabharwal, and B. Aazhang, "On capacity of gaussian 'cheap' relay channel," GLOBECOM, pp. 1776 - 1780, Dec. 2003.

[4] Y. Liang and V.V. Veeravalli., "Gaussian orthogonal relay channel: optimal resource allocation and capacity," Sep. 2005.

[5] J.N. Laneman, D.N.C. Tse, and G.W. Wornell, "Cooperative diversity in wireless networks: Efficient protocols and outage behavior," IEEE Trans. Inform. Theory, vol. 50, pp. 3062 - 3080, Dec. 2004.

[6] R.U. Nabar, H. Bolcskei, and F.W. Kneubuhler, "Fading relay channels: performance limits and space-time signal design," IEEE J. Select. Areas Commun., pp. 1099-1109, Aug. 2004

[7] A. Sendonaris, E. Erkip, and B. Aazhang, "User cooperation diversity. parts i. and ii," IEEE Trans. Commun., vol. 51, pp. 1927 - 1948, Nov. 2003.

[8] A. Stefanov and E. Erkip, "Cooperative coding for wireless networks," IEEE Trans. Commun., vol. 52, pp. 1470 - 1476, Sep. 2004.

[9] M. Janani, A. Hedayat, T.E. Hunter, and A. Nosratinia, "Coded cooperation in wireless communications: space-time transmission and iterative decoding," IEEE Trans. Signal Processing, vol. 52, pp. $362-$ 371, Feb. 2004.

[10] M.A. Khojastepour, N. Ahmed, and B Aazhang, "Code design for the relay channel and factor graph decoding," in Asilomar, 2004, vol. 2, pp. 2000-2004.

[11] A. Chakrabarti, A. de Baynast, A. Sabharwal, and B. Aazhang, "LDPC code design for half-duplex decode-and-forward relaying," in Proc. of the Allerton Conference, Monticello, IL, Sep. 2005.

[12] Zheng Zhang and T. M. Duman, "Capacity approaching turbo coding for half duplex relaying," in Proc. of ISIT, Sep. 2005.

[13] Z. Zhang, I. Bahceci, and T. M. Duman, "Capacity approaching codes for relay channels," in Proc. of ISIT, Jun. 2004.

[14] B. Zhao and M. C. Valenti, "Distributed turbo coded diversity for relay channel," Electronics letters, vol. 39, pp. 786-787, May 2003.

[15] J. Castura and Yongyi Mao, "Rateless coding for wireless relay channels," in Proc. of ISIT, Sep. 2005, pp. 810-814.

[16] G. Kramer, "Communication strategies and coding for relaying," IMA 2005 Summer Program on Wireless Communications.

[17] A. Host-Madsen, "Cooperation in the low power regime," in Allerton, 2004.

[18] D. Marco and D. L. Neuhoff, "Performance of low rate entropyconstrained scalar quantizers," in ISIT, June 2004.

[19] A. Gersho and R. M. Gray, Vector Quantization and Signal Compression, Kluwer Academic Publishers, 1992.

[20] J. Proakis, Digital Communications, McGraw-Hill, 2001.

[21] "ldpcopt - a fast and accurate degree distribution optimizer for ldpc code ensembles," http://lthcwww.epfl.ch/research/ldpcopt/. 